\title{
Taylor Series in Hermitean Clifford Analysis
}

\author{
David Eelbode • Fu Li He
}

Received: 20 February 2009 / Accepted: 7 September 2009 / Published online: 22 September 2009

(C) Birkhäuser Verlag Basel/Switzerland 2009

\begin{abstract}
In this paper, we consider the Taylor decomposition for $h$-monogenic functions in Hermitean Clifford analysis. The latter is to be considered as a refinement of the classical orthogonal function theory, in which the structure group underlying the equations is reduced from $\mathfrak{s o}(2 m)$ to the unitary Lie algebra $\mathrm{u}(m)$.
\end{abstract}

Keywords Hermitean Clifford analysis · Integral transform · Taylor series

Mathematics Subject Classification (2000) Primary 0G35; Secondary 43A65 . $15 \mathrm{~A} 66$

\section{Introduction}

In a series of recent papers [2-4,7], Hermitean Clifford analysis has emerged as a refinement of what is now broadly accepted as classical Clifford analysis. Within this branch of classical analysis, one deals with invariant differential operators for $\operatorname{Spin}(m)$,

Communicated by Frank Sommen.

F. L. He was supported by the China Scholarship Council.

D. Eelbode $(\varangle)$

Department of Mathematics and Computer Science,

University of Antwerp, Campus Middelheim, G-Building,

Middelheimlaan 1, 2020 Antwerpen, Belgium

e-mail: david.eelbode@ua.ac.be; davideelbode@gmail.com

F. L. He

School of Mathematics and Statistics, Wuhan University, 430072 Wuhan, China

e-mail: hefuli999@163.com 
or its Lie algebra $\mathfrak{s o}(m)$, from a function theoretical point of view. We refer to reader to the standard references $[1,6,9]$. From a geometrical point of view, spin-invariant operators can be defined using the Levi-Civita connection $\nabla$ acting on the sections $\phi$ of a suitable (higher) spin bundle on a general Riemannian spin manifold $\mathcal{M}$, see e.g. [13]. The Hermitean refinement of this theory has to be seen as the function theoretical study of the natural operators associated to Kähler geometry for manifolds $\mathcal{M}$ with a complex structure $J$, which reduces the symmetry underlying the system from the spin group to a double cover for the unitary group. It is centred around two complex Hermitean Dirac operators $\underline{\partial}_{z}$ and $\underline{\partial}_{z}^{\dagger}$ which commute with a suitable action of the real algebra $\mathfrak{u}(m)$ (or the complexification $\mathfrak{s l}(m)$ of its semisimple part), realized within the Clifford algebra $\mathbb{R}_{2 m}$.

These differential operators, which can be expressed by means of a complex structure $J \in S O_{\mathbb{R}}(2 m)$ for which $J^{2}=-\mathbf{1}_{2 m}$, refine the complex Laplacian in the sense that $\underline{\partial}_{z} \underline{\partial}_{z}^{\dagger}+\underline{\partial}_{z}^{\dagger} \underline{\partial}_{z}=\Delta_{z}=\sum_{j=1}^{m} \partial_{z_{j}} \partial_{z_{j}^{c}}$, and they lead to a non-elliptic system of equations. Although the first attempts to investigate complex Dirac operators within the setting of Clifford analysis can already be found in e.g. [10-12], a systematic development of the associated function theory and the related questions of invariance w.r.t. the underlying Lie group (and algebra) is still in full progress. The aim of the present paper is to prove a Hermitean generalization of the classical Taylor series in Clifford analysis, which decomposes $h$-monogenic functions in terms of $h$-monogenic polynomial building blocks. Within the function theoretical setting that Clifford analysis provides, this is an important result. To arrive at this result, we will combine techniques coming from representation theory (such as branching rules) and classical analysis (such as the refinement of the Cauchy Theorem).

\section{The Clifford Analysis Setting}

Consider the real orthogonal space $\mathbb{R}^{2 m}$ endowed with the symmetric real-bilinear form $\mathcal{B}_{\mathbb{R}}(\cdot, \cdot)$ of signature $(0,2 m)$, i.e. with $\mathcal{B}_{\mathbb{R}}\left(e_{i}, e_{j}\right)=-\delta_{i j}$. The Lie group $S O_{\mathbb{R}}(2 m)$ consists of orientation preserving automorphisms of $\mathbb{R}^{2 m}$ leaving $\mathcal{B}_{\mathbb{R}}$ invariant. A complex structure $J$ can be defined as a specific $J \in S O_{\mathbb{R}}(2 m)$ satisfying $J^{2}=-\mathbf{1}_{2 m}$. In terms of the standard orthonormal basis $\left(e_{1}, \cdots, e_{2 m}\right)$, we may put $J\left[e_{j}\right]=-e_{j+m}$ and $J\left[e_{j+m}\right]=e_{j}$. Note that this approach can also be reversed: given the triple $(V, \mathcal{B}, J)$, with $V$ a real vector space of dimension $2 m$ and $\mathcal{B}$ a non-degenerate symmetric bilinear form of signature $(0,2 m)$, one can always find a standard $\mathcal{B}$-orthonormal basis such that the previous holds.

In terms of $J \in S O_{\mathbb{R}}(2 m)$, the complex vector space $\mathbb{C}^{2 m}=\mathbb{R}^{2 m} \otimes \mathbb{C}$ endowed with the complex-bilinear form $\mathcal{B}_{\mathbb{C}}$ can be decomposed into a direct sum of two maximally isotropic subspaces $W^{+}$and $W^{-}$. To that end, it suffices to put

$$
\mathbf{1}_{2 m}=\frac{1}{2}\left(\mathbf{1}_{2 m}+i J\right)+\frac{1}{2}\left(\mathbf{1}_{2 m}-i J\right)=\pi^{+}+\pi^{-} .
$$

If we then define $W^{ \pm}=\pi^{ \pm}\left(\mathbb{C}^{2 m}\right)$, it is easily verified that both spaces are isotropic, in the sense that the restrictions of $\mathcal{B}_{\mathbb{C}}$ to $W^{+} \times W^{+}$and $W^{-} \times W^{-}$are identically 
zero, and that $W^{ \pm}$are eigenspaces for the complex-linear map $J_{\mathbb{C}} \in S O(2 m)$ with eigenvalues $\mp i$.

Extending the action of $S O_{\mathbb{R}}(2 m)$ to $\mathbb{C}^{2 m}$, it is clear that the isotropic spaces $W^{ \pm}$ are invariant under the action of those $M \in S O_{\mathbb{R}}(2 m)$ for which $M J=J M$. This prompts the definition of the real Lie group

$$
S O_{J}(2 m)=\left\{M \in S O_{\mathbb{R}}(2 m):[M, J]=0\right\},
$$

whose importance lies in the fact that its action on $W^{ \pm} \times W^{ \pm}$is well-defined and preserves the Hermitean inner product $\mathcal{B}_{\mathbb{C}}$ on $W^{ \pm} \times W^{\mp}$. This essentially says that $S O_{J}(2 m)$ is isomorphic to the (real) unitary Lie group $U(m)$ (for the explicit description of the isomorphism, we refer to [3]).

Let us then consider the complex vector space $\mathbb{C}^{2 m}$ and its Clifford algebra $\mathbb{C}_{2 m}$, generated by $e_{i} e_{j}+e_{j} e_{i}=-2 \delta_{i j}$. As a vector space, $\mathbb{C}_{2 m}$ is isomorphic to a Grassmann algebra and hence inherits a $\mathbb{Z}$-grading into subspaces $\mathbb{C}_{2 m}^{(k)}$ of $k$-vectors. On the other hand, $\mathbb{C}_{2 m}$ is $\mathbb{Z}_{2}$-graded with respect to the main involution, acting on basis vectors by means of $\widetilde{e_{i}}=-e_{i}$ and extending linearly to arbitrary Clifford numbers by $(a b)^{\sim}=\widetilde{a} \widetilde{b}$. This means that $\mathbb{C}_{2 m}=\mathbb{C}_{2 m}^{+} \oplus \mathbb{C}_{2 m}^{-}$can be decomposed into a direct sum of even and odd elements. Within the even part, one can define the (real or complex) spin group $\operatorname{Spin}(2 m)$ which yields a double cover for the (real or complex) group $S O(2 m)$. One e.g. has that

$$
\operatorname{Spin}_{\mathbb{R}}(2 m)=\left\{s=\prod_{j=1}^{2 k} \underline{\omega}_{j}: \underline{\omega}_{j} \in S^{2 m-1}, \quad k \in \mathbb{N}\right\},
$$

where $S^{2 m-1} \subset \mathbb{R}^{2 m}$ denotes the unit sphere containing unit vectors $\underline{\omega}_{j}$ for which $\underline{\omega}_{j}^{2}=-1$. The mapping $\chi: \operatorname{Spin}_{\mathbb{R}}(2 m) \mapsto S O_{\mathbb{R}}(2 m)$ for which $\chi(s)[\underline{X}]=s \underline{X} \bar{s}$ then defines the double cover between both Lie groups. We hereby introduced the main conjugation as an endomorphism of the Clifford algebra, acting on basis vectors by means of $\overline{e_{i}}=-e_{i}$ and extending linearly to arbitrary Clifford numbers by $\overline{a b}=\bar{b} \bar{a}$. On the complex Clifford algebra $\mathbb{C}_{2 m}$, one may formally define the tensor product of the Clifford conjugation with the classical complex conjugation: this is the Hermitean conjugation, denoted by a dagger. This can thus be seen as the complex anti-linear version of the Clifford conjugation, for which $(\lambda a)^{\dagger}=\lambda^{c} \bar{a}$, with $a \in \mathbb{R}_{2 m}$ and $\lambda \in \mathbb{C}$.

Using the projection operators $\pi^{ \pm}$introduced earlier, we can now define the socalled Witt-basis for $\mathbb{C}^{2 m}$ by means of

$$
\mathfrak{f}_{j}=+\pi^{+}\left[e_{j}\right] \text { and } \mathfrak{f}_{j}^{\dagger}=-\pi^{-}\left[e_{j}\right] \quad(1 \leq j \leq m) .
$$

In terms of these Witt basis vectors spanning the vector space $W^{+}$(resp. $W^{-}$), the multiplication rules for $\mathbb{C}_{2 m}$ become

$$
\mathfrak{f}_{j}^{2}=\left(\mathfrak{f}_{j}^{\dagger}\right)^{2}=0 \text { and } \mathfrak{f}_{j} \mathfrak{f}_{k}^{\dagger}+\mathfrak{f}_{k}^{\dagger} \mathfrak{f}_{j}=\delta_{j k} .
$$


Putting $I_{j}=\mathfrak{f}_{j} \mathfrak{f}_{j}^{\dagger}$ and $K_{j}=\mathfrak{f}_{j}^{\dagger} \mathfrak{f}_{j}$, for $1 \leq j \leq m$, we obtain two sets of commuting self-adjoint idempotents, in terms of which $\mathbb{C}_{2 m}=\mathbb{C}_{2 m} \prod_{j=1}^{m}\left(I_{j}+K_{j}\right)$ can be decomposed into a direct sum of $2^{m}$ mutually isomorphic minimal left ideals. The spinor spaces $\mathbb{S}^{ \pm}$carrying the half-integer spin representations for $\mathfrak{s o}(2 m)$ with highest weights $\left(\frac{1}{2}, \ldots, \frac{1}{2}, \pm \frac{1}{2}\right)$ can be defined as each one of these ideals. Classically, this is done in terms of the primitive idempotent $I=I_{1} \cdots I_{m}$. Indeed, $\mathbb{S}^{ \pm}=\mathbb{C}_{2 m}^{ \pm} I=\Lambda_{ \pm}^{\dagger} I$, where the algebra $\Lambda^{\dagger}=\Lambda_{+}^{\dagger} \oplus \Lambda_{-}^{\dagger}$ is the Grassmann algebra generated by the isotropic basis for $W^{-}$. The orthogonal Lie algebra $\mathfrak{s o}(2 m)$ is hereby realized as the Lie algebra $\mathbb{C}_{2 m}^{(2)}$ of bivectors, endowed with the commutator bracket, and acts by Clifford multiplication (from the left) on spinors.

The vector space $\mathbb{R}^{2 m}$ can now be embedded into its Clifford algebra by means of $\underline{X} \hookrightarrow \sum_{j} e_{j} X_{j} \in \mathbb{R}_{2 m}^{(1)}$, and under this identification two fundamental objects in Clifford analysis can be interpreted as endomorphisms on the space of functions with values in the spinor spaces $\mathbb{S}^{ \pm}$, hereby using the fact that $\operatorname{End}(\mathbb{S}) \cong \mathbb{C}_{2 m}$, where $\mathbb{S}=\mathbb{S}^{+} \oplus \mathbb{S}^{-}$. Indeed, both the vector variable $\underline{X}$ and the classical Dirac operator $\underline{\partial}_{X}=\sum_{j} e_{j} \partial_{X_{j}}$ can be seen as multiplication operators on spinor-valued functions on $\mathbb{R}^{2 m}$. The standard theory of Clifford analysis is centred around this Dirac operator, and a fundamental notion is that of a monogenic function $f(\underline{X})$, i.e. a nullsolution for $\underline{\partial}_{X}$.

Two crucial facts in Clifford analysis are the following: the monogenic function theory refines the harmonic function theory, as $-\underline{\partial}_{X}^{2}=\Delta_{2 m}=\sum_{j} \partial_{X_{j}}^{2}$, and $\underline{\partial}_{X}$ commutes with the $\operatorname{Spin}(2 m)$-representation for $\mathbb{S}$-valued functions given by

$$
\begin{aligned}
L: \operatorname{Spin}(2 m) \times \mathcal{C}^{\infty}\left(\mathbb{C}^{2 m}, \mathbb{S}\right) & \mapsto \mathcal{C}^{\infty}\left(\mathbb{C}^{2 m}, \mathbb{S}\right) \\
(s, f(\underline{X})) & \mapsto L(s)[f(\underline{X})]=s f(\bar{s} \underline{X} s),
\end{aligned}
$$

in the sense that $\underline{\partial}_{X} L(s)[f(\underline{X})]=L(s)\left[\underline{\partial}_{X} f(\underline{X})\right]$ for all $s$.

In terms of the Witt basis vectors, we can finally define the basic objects within the Hermitean setting. To that end, it suffices to let the projection operators $\pi^{ \pm}$act on the vector variable $\underline{X} \in \mathbb{R}^{2 m}$ and on the Dirac operator $\underline{\partial}_{X}$, giving rise to two complex vector variables

$$
\begin{aligned}
& \underline{z}=+\pi^{+}[\underline{X}]=\sum_{j} \mathfrak{f}_{j} z_{j} \in W^{+} \\
& \underline{z}^{\dagger}=-\pi^{-}[\underline{X}]=\sum_{j} \mathfrak{f}_{j}^{\dagger} z_{j}^{c} \in W^{-},
\end{aligned}
$$

with $z_{j}=X_{j}+i X_{j+m}, j=1, \cdots, m$, the complex variables associated to the complex structure and two complex Hermitean Dirac operators

$$
\begin{aligned}
& 2 \underline{\partial}_{z}^{\dagger}=+\pi^{+}\left[\underline{\partial}_{X}\right]=2 \sum_{j} \mathfrak{f}_{j} \partial_{z_{j}^{c}} \\
& 2 \underline{\partial}_{z}=-\pi^{-}\left[\underline{\partial}_{X}\right]=2 \sum_{j} \mathfrak{f}_{j}^{\dagger} \partial_{z_{j}},
\end{aligned}
$$

where $\partial_{z_{j}^{c}}$ and $\partial_{z_{j}}$ denote the Cauchy-Riemann operator and its conjugate, in the variable $z_{j}$. Note that $\underline{X}=\underline{z}-\underline{z}^{\dagger}$ with $|\underline{X}|^{2}=\left\{\underline{z}, \underline{z}^{\dagger}\right\}=\underline{z}^{\dagger}+\underline{z}^{\dagger} \underline{z}$ and that $\underline{\partial}_{X}=2\left(\underline{\partial}_{z}^{\dagger}-\underline{\partial}_{z}\right)$ 
with $4\left\{\underline{\partial}_{z}, \underline{\partial}_{z}^{\dagger}\right\}=4 \Delta_{z}=\sum_{j} \partial_{z_{j}} \partial_{z_{j}^{c}}=\Delta_{2 m}$, where the curly brackets denote the anti-commutator. Note also that the Hermitean inner product, preserved by $S O_{J}(2 m)$, can neatly be expressed as $\left\{\underline{z}, \underline{w}^{\dagger}\right\}=\sum_{j} z_{j} w_{j}^{c}$. The central notion in Hermitean Clifford analysis is the following:

Definition 1 A spinor-valued function $F(\underline{X}) \in \mathcal{C}^{1}(\Omega, \mathbb{S})$, with $\Omega \subset \mathbb{R}^{2 m}$ an open subset, is called $h$-monogenic if $\underline{\partial}_{z} F=\underline{\partial}_{z}^{\dagger} F=0$.

The study of these functions and their properties lies at the very heart of the theory: we refer to e.g. [2-4,10,12] for the first results.

\section{Homogeneous Spinor Spaces}

In what follows, projections on the spaces $\mathbb{S}_{j}$ of homogeneous spinors will play a crucial role. Let us therefore have a closer look at these subspaces of $\mathbb{S}$. In the previous section we have found that the (real) unitary Lie group $U(m)$ can be identified with the subgroup $S O_{J}(2 m)$ of the Lie group $S O_{\mathbb{R}}(2 m)$, which means that the real Lie algebra $\mathfrak{u}(m)$ can be realized inside the Lie algebra $\mathbb{R}_{2 m}^{(2)}$, isomorphic to the real compact Lie algebra $\mathfrak{s o}_{\mathbb{R}}(2 \mathrm{~m})$. This was done explicitly in [7], we here only mention the following result:

Definition 2 The complex Lie algebra $\mathfrak{s l}(m)=\mathfrak{s u}(m) \otimes \mathbb{C}$ can be realized as

$$
\mathfrak{s l}(m)=\left\{I_{j}-I_{m}: 1 \leq j \leq m-1\right\} \oplus\left\{\Xi_{j, k}, \Upsilon_{j, k}: 1 \leq j<k \leq m\right\},
$$

where the first summand denotes the Cartan subalgebra $\mathfrak{h}$ for $\mathfrak{s l}(m)$ and where $\Xi_{j, k}=$ $\mathfrak{f}_{j} \mathfrak{f}_{k}^{\dagger}$ (resp. $\Upsilon_{j, k}=\mathfrak{f}_{k} \mathfrak{f}_{j}^{\dagger}$ ) generates the positive root space $\mathfrak{s l}_{L_{j}-L_{k}}$ for each $j<k$ (resp. the negative root space $\mathfrak{s l}_{L_{k}-L_{j}}$ ).

In what follows, we will need to describe function spaces containing Hermitean monogenic polynomials from the point of view of representation theory. To do so, we will consider them as $\mathfrak{s l}(m)$-modules. Finite-dimensional $\mathfrak{s l}(m)$-irreducible modules are described by their highest weight vectors $\underline{\lambda}\left(\lambda_{1}, \cdots, \lambda_{m-1}\right) \in \mathbb{N}^{m-1}$ with $\lambda_{1} \geq \cdots \geq \lambda_{m-1} \geq 0$. In order to describe the fundamental representations as subspaces of the spinor space $\mathbb{S}$, we define the homogeneous spinor spaces:

Definition 3 For all $j \in\{0, \cdots, m\}$, we define the space $\mathbb{S}_{j}$ of homogeneous spinors of degree $j$ as $\mathbb{S}_{j}=\Lambda_{j}^{\dagger} I$, where $\Lambda_{j}^{\dagger} \subset \Lambda^{\dagger}$ is the subspace generated by products of $j$ isotropic vectors $\mathfrak{f}_{k}^{\dagger} \in W^{-}$.

These spaces can also be seen as eigenspaces for the following operator:

Definition 4 The spin-Euler operator is defined as the multiplication operator

$$
\beta:=\sum_{j=1}^{m} K_{j}=\sum_{j=1}^{m} \mathfrak{f}_{j}^{\dagger} \mathfrak{f}_{j} \in \operatorname{End}\left(\mathbb{S}_{j}\right),
$$


acting as the constant $j$ on $\psi_{j} \in \mathbb{S}_{j}$. The projection operator $\pi_{j}: \mathbb{S} \mapsto \mathbb{S}_{j}$ is then given by

$$
\pi_{j}:=\prod_{j \neq k=0}^{m} \frac{\beta-k}{j-k}
$$

The following was proved in e.g. [7]. Note that the subscript in the highest weight denotes how many times an integer is to be repeated:

Theorem 1 For all $1 \leq j \leq m-1$, the spaces $\mathbb{S}_{j}$ are $\mathfrak{s l}(m)$-irreducibles with highest weight $\left(1_{m-j}, 0_{j-1}\right)$, under the multiplicative $\mathfrak{s l}(m)$-action. Both $\mathbb{S}_{0}$ and $\mathbb{S}_{m}$ yield a copy of the trivial representation.

\section{Homogeneous $h$-Monogenic Polynomials}

In view of the fact that we will decompose arbitrary $\mathbb{S}_{j}$-valued functions in terms of $h$-monogenic polynomials, which yield explicit models for irreducible $\mathfrak{s l}(m)$ models within Hermitean Clifford analysis, this section is devoted to their definition and properties.

Definition 5 For any couple of integers $(a, b) \in \mathbb{N} \times \mathbb{N}$, we define the space $\mathcal{P}_{a, b}$ of scalar polynomials which are homogeneous of degree $a$ (resp. $b$ ) in $\underline{z}$ (resp. $\underline{z}^{\dagger}$ ). This means that for all scalars $\lambda, \mu \in \mathbb{C}$,

$$
p_{a, b}\left(\underline{z}, \underline{z}^{\dagger}\right) \in \mathcal{P}_{a, b} \Longrightarrow p_{a, b}\left(\lambda \underline{z}, \mu^{c} \underline{z}^{\dagger}\right)=\lambda^{a}\left(\mu^{c}\right)^{b} p_{a, b}\left(\underline{z}, \underline{z}^{\dagger}\right)
$$

The space of $\mathbb{S}_{j}$-valued $(a, b)$-homogeneous polynomials will be denoted by $\mathcal{P}_{a, b}^{(j)}$. Subspaces containing harmonic (resp. $h$-monogenic) polynomials will be denoted by $\mathcal{H}_{a, b}^{(j)}\left(\operatorname{resp} . \mathcal{M}_{a, b}^{(j)}\right)$.

Note that elements of $\mathcal{P}_{a, b}^{(j)}$ are simultaneous eigenfunctions for the complex Euler operators $\mathbb{E}_{z}$ and $\mathbb{E}_{z}^{\dagger}$, measuring the degree of homogeneity in $\underline{z}$ and $\underline{z}^{\dagger}$, respectively, and the spin-Euler operator $\beta$.

Restricting the (derived) $d L$-action for spinor-valued functions from $\mathfrak{s o}(2 m)$ to $\mathfrak{s l}(m)$, we immediately obtain the action on spaces of polynomials. Recalling the fact that each $H_{j}=I_{j}-I_{m} \in \mathfrak{h}$ acts by means of

$$
d L\left(H_{j}\right)=\left(z_{m} \frac{\partial}{\partial z_{m}}-z_{j} \frac{\partial}{\partial z_{j}}\right)-\left(z_{m}^{c} \frac{\partial}{\partial z_{m}^{c}}-z_{j}^{c} \frac{\partial}{\partial z_{j}^{c}}\right)
$$

on scalar-valued functions, see e.g. [6], one can easily determine the weight space decomposition of polynomial function spaces. We essentially refer to [7] for more detailed calculations, we only mention the key results here. We hereby denote the Cartan product of two irreducible representations by means of $\otimes$. 
Proposition 1 For all $(a, b) \in \mathbb{N}^{2}$ we have the following results for scalar-valued polynomials:

1. Spaces of a-homogeneous polynomials in $\underline{z}$ are symmetric powers of the dual defining representation with highest weight $(a, \ldots, a)$

$$
\mathcal{P}_{a, 0} \cong \operatorname{Sym}^{a}\left(\Lambda^{m-1} \mathbb{C}^{m}\right) \cong \operatorname{Sym}^{a} \mathbb{S}_{1}
$$

2. Spaces of b-homogeneous polynomials in $\underline{z}^{\dagger}$ are symmetric powers of the defining representation with highest weight $(b, 0, \ldots, 0)$

$$
\mathcal{P}_{0, b} \cong \operatorname{Sym}^{b} \mathbb{C}^{m} \cong \operatorname{Sym}^{b} \mathbb{S}_{m-1}
$$

3. Spaces of $(a, b)$-homogeneous harmonic polynomials are irreducible with highest weight $(a+b, a, \ldots, a)$, i.e. $\mathcal{H}_{a, b}=\mathcal{P}_{a, 0} \otimes \mathcal{P}_{0, b}$.

4. The space $\mathcal{P}_{a, b}$ decomposes into harmonic polynomials :

$$
\mathcal{P}_{a, b}=\bigoplus_{j=0}^{\min (a, b)} \mathcal{H}_{a-j, b-j}
$$

In the following section we will use the fact that the space $\mathcal{H}_{k}$ of harmonic polynomials on $\mathbb{R}^{2 m}$, which yields a model for the irreducible $\mathfrak{s o}(2 m)$-module with highest weight $(k, 0, \ldots, 0)$, decomposes into a direct sum of irreducible modules for $\mathfrak{s l}(m)$. Indeed, we have that

$$
\mathcal{H}_{k}=\bigoplus_{a=0}^{k} \mathcal{H}_{a, k-a}
$$

Next, we consider the spaces of $\mathbb{S}_{j}$-valued polynomials. In view of the previous theorem, it suffices to see how the tensor product $\mathcal{H}_{a, b} \otimes \mathbb{S}_{j}$ decomposes. This leads to the Hermitean refinement of the classical monogenic Fischer decomposition, which essentially says that (as $\mathfrak{s o}(2 m)$-modules)

$(k, 0, \ldots, 0) \otimes\left(\frac{1}{2}, \ldots, \frac{1}{2}\right)=\left(\frac{2 k+1}{2}, \frac{1}{2}, \ldots, \frac{1}{2}\right) \oplus\left(\frac{2 k-1}{2}, \frac{1}{2}, \ldots, \frac{1}{2}\right)$.

The Hermitean refinement of this property was proved in [7]. To some extent the proof already appeared in [3], but the latter reference does not address this problem from the point of view of $\mathfrak{s l}(m)$-modules. First of all, we list the degenerate cases:

Proposition 2 In case $a>0$ (resp. $b>0$ ), we have :

$$
\mathcal{H}_{a, b}^{(0)} \cong \underline{z}^{(1)} \mathcal{M}_{a-1, b} \text { and } \mathcal{H}_{a, b}^{(m)} \cong \underline{z}^{\dagger} \mathcal{M}_{a, b-1}^{(m-1)} .
$$


In case $a=0$ or $b=0$ we have

$$
\mathcal{H}_{0, b}^{(0)} \cong \mathcal{M}_{0, b}^{(0)} \text { and } \mathcal{H}_{a, 0}^{(m)} \cong \mathcal{M}_{a, 0}^{(m)}
$$

All other spaces $\mathcal{M}_{a, b}^{(0)}$ and $\mathcal{M}_{a, b}^{(m)}$ are trivial, for $(a, b) \in \mathbb{N}_{0}^{2}$, which is due to the fact that h-monogenic polynomials taking values in $\mathbb{S}_{0}$ or $\mathbb{S}_{m}$ are easily seen to be (anti-)holomorphic in several complex variables.

The general Theorem, decomposing the spaces $\mathcal{H}_{a, b}^{(j)}$ for all other values, can be proved by means of Pieri's formula, which is a representation theoretical description of how to decompose tensor products for $\mathfrak{s l}(m)$-modules. In general, we have that $\mathbb{S}_{j}$-valued harmonic polynomials decompose into at most four irreducible pieces. Before actually giving the Theorem, let us give an example using Young diagrams. Consider e.g. $\mathcal{H}_{2,5} \otimes \mathbb{S}_{2}$ in dimension $m=5$ :
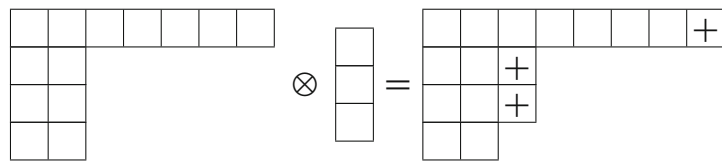

$\bigoplus$
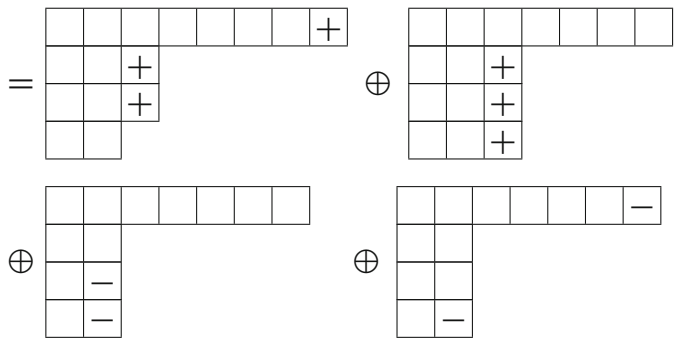

$\bigoplus$

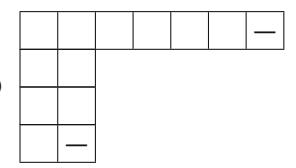

In other words: the first two diagrams are obtained by adding the extra column, either starting from the first or from the second row, whereas the latter two diagrams are obtained by removing the dual of the extra column, starting from the last row, hereby also having the possibility to remove a box from the first row. If the column corresponding to the module $\mathbb{S}_{m-j}$ has $j$ boxes, the dual column (referring to the dual module $\left.\mathbb{S}_{j}\right)$ has $(m-j)$ boxes.

Theorem 2 Suppose $H_{a, b}^{(j)} \in \mathcal{H}_{a, b}^{(j)}$ with $0<j<m$ and $0<a$, b. Then $H_{a, b}^{(j)}$ decomposes as

$$
H_{a, b}^{(j)}=M_{a, b}^{(j)}+\underline{z} M_{a-1, b}^{(j+1)}+\underline{z}^{\dagger} M_{a, b-1}^{(j-1)}+\left(c_{1} \underline{z} \underline{z}^{\dagger}+c_{2} \underline{z}^{\dagger} \underline{z}\right) M_{a-1, b-1}^{(j)},
$$

where all polynomials $M_{a, b}^{(j)} \in \mathcal{M}_{a, b}^{(j)}$ are given by

$$
\begin{aligned}
M_{a-1, b}^{(j+1)}\left(\underline{z}, \underline{z}^{\dagger}\right) & =+\left(\underline{\partial}_{z}+\frac{1}{c_{2}} \underline{z}^{\dagger} \underline{\partial}_{z}^{\dagger} \underline{\partial}_{z}\right) \frac{H_{a, b}^{(j)}\left(\underline{z}, \underline{z}^{\dagger}\right)}{c_{1}+1} \\
M_{a, b-1}^{(j-1)}\left(\underline{z}, \underline{z}^{\dagger}\right) & =-\left(\underline{\partial}_{z}^{\dagger}-\frac{1}{c_{1}} \underline{z} \underline{\partial}_{z} \underline{\partial}_{z}^{\dagger}\right) \frac{H_{a, b}^{(j)}\left(\underline{z}, \underline{z}^{\dagger}\right)}{c_{2}-1} \\
M_{a-1, b-1}^{(j)}\left(\underline{z}, \underline{z}^{\dagger}\right) & =-\frac{\underline{\partial}_{z}^{\dagger} \underline{\partial}_{z}}{\left(1+c_{1}-c_{2}\right) c_{1} c_{2}} H_{a, b}^{(j)}\left(\underline{z}, \underline{z}^{\dagger}\right),
\end{aligned}
$$


with $c_{1}=(a+j-1)$ and $c_{2}=-(b+m-j-1)$. Note that these constants can also be replaced by the differential operators $\left(\beta+\mathbb{E}_{z}\right)$ and $\left(m-\beta+\mathbb{E}_{z}^{\dagger}\right)$, acting as the desired constant on the space $\mathcal{M}_{a, b}^{(j)}$.

For future purposes, we will give each of these projection operators its own symbol:

$$
\begin{aligned}
\pi_{a, b ; j}^{+}: \mathcal{H}_{a, b}^{(j)}\left(\underline{z}, \underline{z}^{\dagger}\right) \mapsto \mathcal{M}_{a-1, b}^{(j+1)} \\
\pi_{a, b ; j}^{-}: \mathcal{H}_{a, b}^{(j)}\left(\underline{z}, \underline{z}^{\dagger}\right) \mapsto \mathcal{M}_{a, b-1}^{(j-1)} \\
\pi_{a, b ; j}^{0}: \mathcal{H}_{a, b}^{(j)}\left(\underline{z}, \underline{z}^{\dagger}\right) \mapsto \mathcal{M}_{a-1, b-1}^{(j)},
\end{aligned}
$$

where the upper indices indicate whether the index $j$ has to be raised/lowered or stays the same, and where the lower indices $(a, b ; j)$ characterize the domain space $\mathcal{H}_{a, b} \otimes \mathbb{S}_{j}$. The explicit definition for these projection operators follows from the previous theorem.

Remark 1 In case $j \in\{1, m-1\}$, one of these monogenic polynomials will turn out to be identically zero, such that we end up with three summands only. Recalling the fact that the Young diagrams can have at most $(m-1)$ rows, this is also clear from the approach involving Young diagrams.

Remark 2 An alternative proof for the Hermitean Fischer decomposition can be given in terms of the Howe dual pair $U(m) \times \mathfrak{g l}(m)$, see [5].

\section{Taylor Expansions for Hermitean Monogenics}

In this section, we will derive a Taylor expansion for Hermitean monogenic functions $F(\underline{X})$. Let us therefore introduce the following definition:

Definition 6 Let $\Omega \subset \mathbb{R}^{2 m}$ be an open subset. We then define the space of $\mathbb{S}$-valued $h$-monogenic functions in $\Omega$ by means of

$$
\mathcal{M}_{h}(\Omega, \mathbb{S})=\left\{F(\underline{X}) \in \mathcal{C}^{1}(\Omega, \mathbb{S}): \underline{\partial}_{z} F=\underline{\partial}_{z}^{\dagger} F=0\right\} .
$$

The subspace containing $\mathbb{S}_{j}$-valued functions will be denoted by $\mathcal{M}_{h}^{(j)}(\Omega)$.

Let $F(\underline{X}) \in \mathcal{M}_{h}^{(j)}(\Omega)$ be an $\mathbb{S}_{j}$-valued $h$-monogenic function on $\Omega$. In order to derive its Hermitean monogenic Taylor expansion, we will start from the classical (orthogonal) Cauchy formula. In view of the fact that $\mathcal{M}_{h} \subset \mathcal{C}^{1}(\Omega) \cap \operatorname{ker}\left(\underline{\partial}_{X}\right)$, we clearly have for all $\underline{X} \in \Omega$ that

$$
F(\underline{X})=\int_{\partial B(R)} E(\underline{Y}-\underline{X}) d \sigma_{Y} f(\underline{Y}),
$$

where $B(R) \subset \Omega$ denotes the ball with radius $R>|\underline{X}|$ lying completely inside $\Omega$. The classical Taylor expansion is usually derived from the decomposition of the Cauchy 
kernel into Gegenbauer polynomials, see e.g. [6]. Putting $\underline{X}=|\underline{X}| \underline{\omega}$ and $\underline{Y}=|\underline{Y}| \underline{\eta}$, we have that

$$
\begin{aligned}
E(\underline{Y}-\underline{X}) & =-\frac{1}{A_{2 m}(2 m-2)} \underline{\partial}_{X} \frac{1}{|\underline{X}-\underline{Y}|^{2 m-2}} \\
& =-\frac{1}{A_{2 m}(2 m-2)} \underline{\partial}_{X} \sum_{l=0}^{\infty} \frac{|\underline{X}|^{l}}{|\underline{Y}|^{l+2 m-2}} C_{l}^{m-1}(t),
\end{aligned}
$$

where $A_{2 m}$ denotes the surface area of the unit sphere $S^{2 m-1} \subset \mathbb{R}^{2 m}$ and where $C_{b}^{a}(t)$ denotes a Gegenbauer polynomial in the real variable $t=\langle\underline{\omega}, \underline{\eta}\rangle$. Note also that this series converges normally in closed balls $\bar{B}(r)$, with $r<|\underline{Y}|$, and that each term in the series is a scalar-valued harmonic polynomial of degree $l$ in $\underline{X}$. This means that the branching rule (1) can be applied to decompose this harmonic polynomial $H_{l}(\underline{X})=|\underline{X}|^{l} C_{l}^{m-1}(t)$ into a direct sum of $(l+1)$ harmonic polynomials $H_{a, l-a}\left(\underline{z}, \underline{z}^{\dagger}\right)$ belonging to an irreducible representation space for the algebra $\mathfrak{s l}(\mathrm{m})$.

This particular branching of the classical Gegenbauer polynomial is described, from the representation theoretical point of view, in e.g. [14]. In recent work [8], we have shown how to obtain the resulting special functions using Clifford analysis techniques only. For the sake of completeness, we will briefly describe this procedure here. The main observation is that the function $|\underline{X}|^{k} C_{k}^{m-1}|\underline{Y}|^{k}$ defines a so-called zonal harmonic polynomial in both variables $\underline{X}$ and $\underline{Y}$, where the term 'zonal' refers to the fact that this polynomial essentially depends on the inner product between the variables $\underline{X}$ and $\underline{Y}$. In view of our definition for a Hermitean inner product on the isotropic subspaces $W^{ \pm}$, which can be written in terms of an anti-commutator, a good generalization to the Hermitean case is given by the following notion:

Definition 7 A function $F(\underline{X}, \underline{Y}): \mathbb{R}^{2 m} \times \mathbb{R}^{2 m} \mapsto \mathbb{C}$ is called zonal Hermitean if it depends on the inner products $\left\{\underline{z}, \underline{w}^{\dagger}\right\},\{\underline{z} \dagger, \underline{w}\},\left\{\underline{z}, \underline{z}^{\dagger}\right\}$ and $\left\{\underline{w}, \underline{w}^{\dagger}\right\}$, where we have put $\underline{X}=\underline{z}-\underline{z}^{\dagger}$ and $\underline{Y}=\underline{w}-\underline{w}^{\dagger}$.

The most convenient generalization of the (orthogonal) scalar zonal variable $t$ turned out to be the Hermitean scalar zonal variable $s$ given by

$$
s=\frac{\left\{\underline{z}, \underline{w}^{\dagger}\right\}\left\{\underline{z}^{\dagger}, \underline{w}\right\}}{\left\{\underline{z}, \underline{z}^{\dagger}\right\}\left\{\underline{w}, \underline{w}^{\dagger}\right\}},
$$

which means that the canonically defined generalization of the classical Gegenbauer equation, leading to Hermitean zonal harmonic polynomials which are $(k, l)$ homogeneous in $\underline{z}$ and $\underline{z}^{\dagger}$ (resp. $\underline{w}^{\dagger}$ and $\underline{w}$ ), is given by (e.g. for $k \geq l$ ):

$$
\Delta_{z}\left(\left\{\underline{z}, \underline{w}^{\dagger}\right\}^{k-l}\left\{\underline{z}, \underline{z}^{\dagger}\right\}^{l}\left\{\underline{w}, \underline{w}^{\dagger}\right\}^{l} S_{k, l}(s)\right)=0 .
$$

This leads to the following hypergeometric differential equation for $S_{k, l}(s)$ :

$$
s(1-s) S_{k, l}^{\prime \prime}+((1+k-l)-(m+k-l) s) S_{k, l}^{\prime}+l(k+m-1) S_{k, l}=0,
$$


whose polynomial solution

$$
S_{k, l}(s)=F(k+m-1,-l ; 1+k-l ; s)
$$

can be expressed in terms of the Jacobi polynomials $P_{k}^{(\alpha, \beta)}(t)$. The fact that these polynomials indeed give the proper generalization of the classical zonal polynomials is confirmed by the following branching formulae:

Proposition 3 Under the branching from $\mathfrak{s o}(2 m)$ to $\mathfrak{s l}(m)$, the classical (zonal) Gegenbauer polynomials decompose as:

$$
|\underline{X}|^{l}|\underline{Y}|^{l} C_{l}^{m-1}(t)=\sum_{q=0}^{l} C_{q, l-q}^{m-1}\left(\underline{z}, \underline{z^{\dagger}} ; \underline{w}, \underline{w} \underline{w}^{\dagger}\right) \in \bigoplus_{q=0}^{l} \mathcal{H}_{q, l-q}
$$

where the harmonic polynomials $C_{q, l-q}^{m-1}\left(\underline{z}, \underline{z}^{\dagger} ; \underline{w}, \underline{w}^{\dagger}\right)$ are explicitly given by:

(i) in case $l=2 p$ is even:

$$
\begin{aligned}
& C_{p+q, p-q}^{m-1}=c_{p, q}\left(\left\{\underline{z}, \underline{z}^{\dagger}\right\}\left\{\underline{w}, \underline{w}^{\dagger}\right\}\right)^{p-q}\left\{\underline{z}, \underline{u}^{\dagger}\right\}^{2 q} S_{p+q, p-q}(s) \\
& C_{p-q, p+q}^{m-1}=c_{p, q}\left(\left\{\underline{z}, \underline{z}^{\dagger}\right\}\left\{\underline{w}, \underline{w}^{\dagger}\right\}\right)^{p-q}\left\{\underline{z}^{\dagger}, \underline{u}\right\}^{2 q} S_{p+q, p-q}(s),
\end{aligned}
$$

with $0 \leq q \leq p$ and where we have introduced the constants

$$
c_{p, q}=(-1)^{p-q} \frac{(p+q+m-2) !}{(p-q) !(m-2) !(2 q) !} .
$$

(i) in case $l=2 p+1$ is odd:

$$
\begin{aligned}
& C_{1+p+q, p-q}^{m-1}=d_{p, q}\left(\left\{\underline{z}, \underline{z}^{\dagger}\right\}\left\{\underline{w}, \underline{w}^{\dagger}\right\}\right)^{p-q}\left\{\underline{z}, \underline{w}^{\dagger}\right\}^{2 q+1} S_{1+p-q, p-q}(s) \\
& C_{p-q, 1+p+q}^{m-1}=d_{p, q}\left(\left\{\underline{z}, \underline{z}^{\dagger}\right\}\left\{\underline{w}, \underline{w}^{\dagger}\right\}\right)^{p-q}\left\{\underline{z^{\dagger}}, \underline{w}\right\}^{2 q+1} S_{1+p-p, p-q}(s),
\end{aligned}
$$

with $0 \leq q \leq p$ and where we have introduced the constants

$$
d_{p, q}=(-1)^{p-q} \frac{(p+q+m-1) !}{(p-q) !(m-2) !(2 q+1) !} .
$$

Proof Let us prove for example the statement for $l=2 p$, the other statement is then proved in a completely similar way. Invoking the identity

$$
C_{2 p}^{m-1}(t)=(-1)^{p}\left(\begin{array}{c}
p+m-2 \\
p
\end{array}\right) F\left(-p, p+m-1 ; \frac{1}{2} ; t^{2}\right)
$$


for the Gegenbauer polynomial of even degree $l=2 p$ and recalling the fact that the Euclidean inner product satisfies $2\langle\underline{X}, \underline{Y}\rangle=\left\{\underline{z}, \underline{w}^{\dagger}\right\}+\{\underline{z} \dagger, \underline{w}\}$, we get that

$$
|\underline{X}|^{2 p}|\underline{Y}|^{2 p} C_{2 p}^{m-1}(t)=\sum_{j=0}^{p} \gamma_{j ; p}\left(\left\{\underline{z}, \underline{z^{\dagger}}\right\}\left\{\underline{w}, \underline{w^{\dagger}}\right\}\right)^{p-j} \sum_{i=0}^{2 j}\left(\begin{array}{c}
2 j \\
i
\end{array}\right)\left\{\underline{z}, \underline{w}^{\dagger}\right\}^{i}\left\{\underline{w}, \underline{z}^{\dagger}\right\}^{2 j-i}
$$

where we have temporarily introduced the shorthand notation

$$
\gamma_{j ; p}=(-1)^{p}\left(\begin{array}{c}
p+m-2 \\
p
\end{array}\right) \frac{(-p)_{j}(p+m-1)_{j}}{2^{2 j} j !\left(\frac{1}{2}\right)_{p}} .
$$

Next, putting $q=j-i$, it is clear that the second summation can be written as

$$
\begin{aligned}
\sum_{i=0}^{2 j}\left(\begin{array}{c}
2 j \\
i
\end{array}\right)\left\{\underline{z}, \underline{w}^{\dagger}\right\}^{i}\left\{\underline{w}, \underline{z}^{\dagger}\right\}^{2 j-i}=\left(\begin{array}{c}
2 j \\
j
\end{array}\right) s^{j}\left(\left\{\underline{z}, \underline{z^{\dagger}}\right\}\left\{\underline{w}, \underline{w}^{\dagger}\right\}\right)^{j} \\
\quad+\sum_{i=0}^{j-1}\left(\begin{array}{c}
2 j \\
i
\end{array}\right)\left(s^{i}\left(\left\{\underline{z}, \underline{z}^{\dagger}\right\}\left\{\underline{w}, \underline{w}^{\dagger}\right\}\right)^{p-q}\left(\left\{\underline{z}, \underline{w}^{\dagger}\right\}^{2 q}+\left\{\underline{z} \underline{z}^{\dagger}, \underline{w}\right\}^{2 q}\right)\right) .
\end{aligned}
$$

Invoking some elementary identities for the Gamma function and recalling the definition for the coefficients in a hypergeometric series, the first term in the left hand side is easily seen to simplify as follows:

$$
\left(\left\{\underline{z}, \underline{z}^{\dagger}\right\}\left\{\underline{w}, \underline{w}^{\dagger}\right\}\right)^{p} \sum_{j=0}^{p} \gamma_{j}(p)\left(\begin{array}{c}
2 j \\
j
\end{array}\right) s^{j}=c_{p, 0} F(-p, p+m-1 ; 1 ; s) .
$$

A similar thing can be done for the other summations above, hereby using the fact that a new labeling of indices leads to

$$
\sum_{j=1}^{l} \sum_{i=0}^{j-1}=\sum_{q=1}^{l} \sum_{i=0}^{l-q}
$$

and that the (formal) quotient of singularities in $\gamma_{j}(p)$ can be simplified as

$$
\frac{\Gamma(q-p)}{\Gamma(-p)}=(-1)^{q} \frac{p !}{(p-q) !},
$$

hereby using the fact that $\Gamma(z) \Gamma(1-z) \sin (\pi z)=\pi$.

Applying this branching to the Cauchy kernel (2), and invoking the fact that the surface element on the boundary $\partial B(R)$ is given by $d \sigma_{Y}=R^{2 m-1} \underline{\eta} d \underline{\eta}$, it is clear that 
Cauchy's theorem yields:

$$
F(\underline{X})=\frac{-1}{2(m-1) A_{2 m}} \sum_{l=0}^{\infty} \sum_{q=0}^{l} \frac{1}{R^{2 l-1}} \int_{S^{2 m-1}} \underline{\partial}_{X} C_{q, l-q}^{m-1}\left(\underline{z}, \underline{z} ; \underline{w}, \underline{w}^{\dagger}\right) \underline{\eta} F(R \underline{\eta}) d \underline{\eta},
$$

for any $F \in \mathcal{M}_{h}^{(j)}(\Omega)$, with $B(R) \subset \Omega$ and $|\underline{X}|<R$. Let us then introduce the following notation:

$$
\underline{\eta}=\frac{\underline{Y}}{|\underline{Y}|}=\frac{\underline{w}-\underline{w}^{\dagger}}{|\underline{Y}|}=\underline{\eta}_{w}-\underline{\eta}_{w}^{\dagger}
$$

Remark Note that $\underline{\eta}_{w} \in W^{+}$and $\underline{\eta}_{w}^{\dagger} \in W^{-}$are no unit vectors in the classical sense. Indeed, whereas $\underline{\eta} \in S^{2 m-1}$ is homogeneous of degree 0 in the variable $\underline{Y}$, the former vectors are homogeneous of degree $(0,-1)$ and $(-1,0)$, respectively.

In view of the fact that $F(\underline{X})$ is $\mathbb{S}_{j}$-valued, it is clear that as a polynomial in $\left(\underline{z}, \underline{z}^{\dagger}\right)$ we have:

$$
C_{q, l-q}^{m-1}\left(\underline{z}, \underline{z}^{\dagger} ; \underline{w}, \underline{w}^{\dagger}\right)\left(\underline{\eta}_{w}-\underline{\eta}_{w}^{\dagger}\right) F(R \underline{\eta}) \in \mathcal{H}_{q, l-q}^{(j-1)} \bigoplus \mathcal{H}_{q, l-q}^{(j+1)}
$$

Applying theorem 2, both summands can then for each $q \in\{0,1, \cdots, l\}$ be decomposed into a direct sum of (at most) four $h$-monogenic polynomials:

$$
\begin{aligned}
& \mathcal{H}_{q, l-q}^{(j-1)}=\left\{\mathcal{M}_{q, l-q}^{(j-1)}+\left(\begin{array}{c}
\underline{z} \mathcal{M}_{q-1, l-q}^{(j)} \\
\underline{z}^{\dagger} \mathcal{M}_{q, l-q-1}^{(j-2)}
\end{array}\right)+\left(C_{1} \underline{z} \underline{z}^{\dagger}+C_{2} \underline{z}^{\dagger} \underline{z}\right) \mathcal{M}_{q-1, l-q-1}^{(j-1)}\right\} \\
& \mathcal{H}_{q, l-q}^{(j+1)}=\left\{\mathcal{M}_{q, l-q}^{(j+1)}+\left(\begin{array}{c}
\underline{z} \mathcal{M}_{q-1, l-q}^{(j+2)} \\
\underline{z}^{\dagger} \mathcal{M}_{q, l-q-1}^{(j)}
\end{array}\right)+\left(C_{1}^{\prime} \underline{z} \underline{z}^{\dagger}+C_{2}^{\prime} \underline{z}^{\dagger} \underline{z}\right) \mathcal{M}_{q-1, l-q-1}^{(j+1)}\right\},
\end{aligned}
$$

where all constants depend on $(j, q, l)$. Note however that we will not need all of them, in view of the following argument: because the left-hand side of the Cauchy formula contains an $\mathbb{S}_{j}$-valued function, the right-hand side should be projected onto this homogeneous spinor space as well. Recalling the fact that $\underline{\partial}_{X}=2\left(\underline{\partial}_{z}^{\dagger}-\underline{\partial}_{z}\right)$, it is immediately clear that

$$
\underline{\partial}_{X} \mathcal{H}_{q, l-q}^{(j-1)} \stackrel{\pi_{j}}{\longrightarrow}\left(\begin{array}{c}
2(j+q-1) \mathcal{M}_{q-1, l-q}^{(j)} \\
+ \\
2(j+q-2)(l+m-1) \underline{z}^{\dagger} \mathcal{M}_{q-1, l-q-1}^{(j-1)}
\end{array}\right)
$$


and

$$
\underline{\partial}_{X} \mathcal{H}_{q, l-q}^{(j+1)} \stackrel{\pi_{j}}{\longrightarrow}\left(\begin{array}{c}
-2(m-j+l-q-1) \mathcal{M}_{q, l-q-1}^{(j)} \\
+ \\
2(m-j+l-q-2)(l+m-1) \underline{z}_{\mathcal{M}_{q-1, l-q-1}^{(j+1)}}^{(m-1)}
\end{array}\right)
$$

At the same time, we get that the projections on the other homogeneous spinor-parts lead to an identity which gives zero. Let us then focus on the polynomial building blocks in terms of which arbitrary Hermitean monogenic functions $F(\underline{X}) \in \mathcal{M}_{h}^{(j)}(\Omega)$ decompose. They clearly come in four different families, which we label as follows (using the projection operators from Theorem 2):

$$
\begin{aligned}
& \mathcal{F}_{(j)}^{+}:=\left\{\pi_{q, l-q ; j-1}^{+}\left(C_{q, l-q}^{m-1}\left(\underline{z}, \underline{z}^{\dagger} ; \underline{w}, \underline{w}^{\dagger}\right) \underline{\eta}_{w} F(R \underline{\eta})\right): 0 \leq q \leq l \in \mathbb{N}\right\} \\
& \mathcal{F}_{(j)}^{-}:=\left\{\pi_{q, l-q ; j+1}^{-}\left(C_{q, l-q}^{m-1}\left(\underline{z}, \underline{z}^{\dagger} ; \underline{w}, \underline{w}^{\dagger}\right) \underline{\eta}_{w}^{\dagger} F(R \underline{\eta})\right): 0 \leq q \leq l \in \mathbb{N}\right\} .
\end{aligned}
$$

Both families contain homogeneous $h$-monogenic polynomials, which follows from Theorem 2, and clearly form the analogue of the (classical) $k$-monogenic summands in the Taylor expansion for a monogenic function in the orthogonal framework. Apart from these, there is however a second type of contributions which has a different nature. Indeed, introducing the families

$$
\begin{aligned}
& \mathcal{G}_{(j)}^{+}:=\left\{\underline{z}^{\dagger} \pi_{q, l-q ; j-1}^{0}\left(C_{q, l-q}^{m-1}\left(\underline{z}, \underline{z}^{\dagger} ; \underline{w}, \underline{w}^{\dagger}\right) \underline{\eta}_{w} F(R \underline{\eta})\right): 0 \leq q \leq l \in \mathbb{N}\right\} \\
& \mathcal{G}_{(j)}^{-}:=\left\{\underline{z}_{q, l-q ; j+1}^{0}\left(C_{q, l-q}^{m-1}\left(\underline{z}, \underline{z}^{\dagger} ; \underline{w}, \underline{w}^{\dagger}\right) \underline{\eta}_{w}^{\dagger} F(R \underline{\eta})\right): 0 \leq q \leq l \in \mathbb{N}\right\},
\end{aligned}
$$

we clearly get two types of contributions consisting of polynomials which are not $h$-monogenic themselves. This differs greatly from the classical orthogonal case, where each building block in the Taylor expansion is monogenic with respect to the Dirac operator. However, this somewhat odd behaviour reflects a similar peculiarity on the level of reproducing integral formulae: whereas monogenic functions can be obtained through Cauchy's formula, expressed in terms of a fundamental solution which is monogenic itself, $h$-monogenic functions have an integral representation which can be seen as a generalization of the Martinelli-Bochner formula. The most important feature distinguishing this kernel from its orthogonal version (the Cauchy kernel), is the fact that it is not $h$-monogenic.

Putting the pieces of the puzzle together, we have thus proved the following result, which can be seen as the Hermitean generalization of the orthogonal Taylor decomposition:

Theorem 3 Given an arbitrary function $F \in \mathcal{M}_{h}^{(j)}(\Omega)$, there exist four series of h-monogenic polynomials (with $0 \leq q \leq l$ and $l \in \mathbb{N}$ )

$$
\left(F_{(j ; q, l)}^{ \pm}\right)_{q, l} \subset \mathcal{F}_{(j)}^{ \pm} \text {and }\left(G_{(j ; q, l)}^{ \pm}\right)_{q, l} \subset \mathcal{G}_{(j)}^{ \pm},
$$


such that for all $|\underline{X}|<R$, with $B(R) \subset \Omega$, we have

$$
F(\underline{X})=-\frac{1}{2(m-1) A_{m}} \sum_{l=0}^{\infty} \sum_{q=0}^{l} \frac{1}{R^{2 l-1}}\left(\begin{array}{c}
F_{(j ; q, l)}^{+}+\underline{z}^{\dagger} G_{(j ; q, l)}^{+} \\
F_{(j ; q, l)}^{-}+\underline{z} G_{(j ; q, l)}^{-}
\end{array}\right) .
$$

These monogenic functions are, respectively, given by the following integrals:

$$
\begin{aligned}
F_{(j ; q, l)}^{+} & =2(j+q-1) \int \pi_{q, l-q ; j-1}^{+} C_{q, l-q}^{m-1} \underline{\eta}_{w} F(R \underline{\eta}) d \underline{\eta} \\
F_{(j ; q, l)}^{-} & =-2(m-j+l+q-1) \int \pi_{q, l-q ; j+1}^{-} C_{q, l-q}^{m-1} \underline{\eta}_{w}^{\dagger} F(R \underline{\eta}) d \underline{\eta} \\
G_{(j ; q, l)}^{+} & =2(j+q-2)(l+m-1) \int \pi_{q, l-q ; j-1}^{0} C_{q, l-q}^{m-1} \underline{\eta}_{w} F(R \underline{\eta}) d \underline{\eta} \\
G_{(j ; q, l)}^{-} & =-2(m-j+l+q-2)(l+m-1) \int \pi_{q, l-q ; j+1}^{0} C_{q, l-q}^{m-1} \underline{\eta}_{w}^{\dagger} F(R \underline{\eta}) d \underline{\eta}
\end{aligned}
$$

and the series (9) converges normally on each $\bar{B}(r)$ with $r<R$.

\section{References}

1. Brackx, F., Delanghe, R., Sommen, F.: Clifford Analysis, Research Notes in Mathematics 76, Pitman, London (1982)

2. Brackx, F., De Schepper, H., Sommen, F.: A hermitean setting for wavelet analysis: the basics. In: Proceedings of the 4th International Conference on Wavelet Analysis and its Applications, University of Macau, China (2005)

3. Brackx, F., Bureš, J., De Schepper, H., Eelbode, D., Sommen, F., Souček, V.: Fundaments of Hermitean Clifford analysis-Part I: complex structure. Complex Anal. Oper. Theory 1, 341-365 (2007)

4. Brackx, F., Bureš, J., De Schepper, H., Eelbode, D., Sommen, F., Souček, V.: Fundaments of Hermitean Clifford analysis-Part II: splitting of $h$ - monogenic equations. Complex Var. Ell. Equ. 52, 1063-1079

5. Brackx, F., De Schepper, H., Eelbode, D., Souček, V.: Howe dual pairs in Hermitean Clifford analysis. Revista Matematica IberoAmericana (2009, accepted)

6. Delanghe, R., Sommen, F., Souček, V.: Clifford Analysis and Spinor Valued Functions. Kluwer, Dordrecht (1992)

7. Eelbode, D.: Irreducible $\mathfrak{s l}(m)$ - modules of Hermitean monogenics. Complex Var. Ell. Equ. 53(10), 975-987 (2008)

8. Eelbode, D.: Zonal Hermitean Monogenic Functions, to appear In: Proceedings of the 15th ICFIDCAA, Osaka, Japan (2007)

9. Gilbert, J., Murray, M.A.M.: Clifford Algebras and Dirac Operators in Harmonic Analysis. Cambridge University Press, Cambridge (1991)

10. Rocha-Chavez, R., Shapiro, M., Sommen, F.: Integral theorems for functions and differential forms in $\mathbb{C}^{m}$, Research Notes in Math. 428, Chapman and Hall / CRC Press, Boca Raton, London (2001)

11. Ryan, J.: Complexified Clifford analysis. Complex Var. 1, 119-149 (1982)

12. Sabadini, I., Sommen, F.: Hermitian Clifford analysis and resolutions, MMAS vol. 25, nos. 16-18. In: Sommen, F., Sproessig, W. (eds.) Special Issue: Clifford Analysis and Applications, pp. 1395-1414 (2002)

13. Stein, E.W., Weiss, G.: Generalization of the Cauchy-Riemann equations and representations of the rotation group. Am. J. Math. 90, 163-196 (1968)

14. Vilenkin, N.Ja., Klimyk, A.U.: Representations of Lie Groups and Special Functions, vol. 2. Kluwer, Dordrecht (1993) 